\title{
EXCHANGE INTERACTION IN ZnCrSe SEMIMAGNETIC SEMICONDUCTOR*
}

\author{
W. Mac, Nguyen The Khol ${ }^{\dagger}$, A. Twardowski, J.A. GaJ \\ Institute of Experimental Physics, Warsaw University \\ Hoża 69, 00-681 Warszawa, Poland
}

and M. Demianiuk

Institute of Technical Physics, W.A.T., Warszawa, Poland

\begin{abstract}
Magnetoreflectance and magnetization of $\mathrm{ZnCrSe}$ were measured for $B \leq 5 \mathrm{~T}$ and $T=2 \mathrm{~K}$. A linear dependence between exciton splitting and magnetization was found. The $s, p-d$ exchange parameter was estimated: $N_{0} \alpha-N_{0} \beta=0.67 \mathrm{eV}$.
\end{abstract}

PACS numbers: 75.50.Pp

The most popular semimagnetic or diluted magnetic semiconductors (DMS) are materials with $\mathrm{Mn}, \mathrm{Co}$ and $\mathrm{Fe}$ as the magnetic ions [1]. In all of them a strong exchange interaction between magnetic ions and band carriers has been observed. It seems rather well established that in these materials the $p-d$ exchange results mostly from the hybridization of $d$ orbitals ( $3 t$-type and $2 e$-type) with the valence band. In the centre of the Brillouin zone only the $t$-orbitals hybridize with the valence band. From this point of view the situation for $\mathrm{Mn}-, \mathrm{Co}-$ and Fe-DMS is similar, since for all of them $t$-orbitals are singly occupied (Mn: $d^{5}=$ $t_{+}^{3} e_{+}^{2}$, Fe: $\left.d^{6}=t_{+}^{3} e_{+}^{2} e_{-}^{1}, \mathrm{Co}: d^{7}=t_{+}^{3} e_{+}^{2} e_{-}^{2}\right)$. In such a case, a simple isotropic Heisenberg Hamiltonian constitutes a good approximation for the $p-d$ exchange [2], and band splittings should be parametrized by macroscopic magnetization. In fact, such behaviour was encountered in the experiment [1]. The situation for Cr-DMS is different: two $t$ orbitals are occupied and the remaining one is empty $\left(d^{4}=t_{+}^{2} e_{+}^{2}\right)$. In this case non-Heisenberg terms in the Hamiltonian are expected [2, 3]. In view of that, we studied newly grown $\mathrm{ZnCrSe}$, with $\mathrm{Cr}$ concentration $x<$ 0.005 . Magnetoreflectivity from the (110) surface was measured in the range of the fundamental absorption edge at $T=2 \mathrm{~K}$ and an exciton line was observed. In the presence of a magnetic field ( $B=0 \div 5 \mathrm{~T}$, Faraday configuration) the exciton line splits into two components (each visible in one of the circular polarizations). We recall that for other DMS the exciton line splits into four components [1]: lines $\mathrm{A}, \mathrm{B}$ ( $\sigma^{+}$polarization) and $\mathrm{C}, \mathrm{D}$ ( $\sigma^{-}$polarization). We believe that due to

*Supported by the the Committee for Scientific Research (grant nr 202239101 ).

tOn leave from Pedagogical University, Hanoi 
small splittings and rather large width of the exciton lines, the lines in the same polarization are not resolved in our case. Therefore, the $\sigma^{+}\left(\sigma^{-}\right)$exciton line is composed of unresolved lines $A$ and $B$ (C and D). Since line A (D) is 3 times stronger than line B (C), we assume that the observed exciton line represents mostly line A (D). The Zeeman splittings observed in this work exhibit opposite sign to that usually reported in DMS (we observed that $\sigma^{+}$component at a higher energy than the $\sigma^{-}$component).

Magnetization (measured on the same samples on which optical work was done) shows typical paramagnetic behaviour. It was found to obey a crystal field model calculation [3] performed for isolated ions. Cr mole fraction values obtained from fitting the absolute magnetization are indicated in Fig. 1. The form of the magnetization does not follow the Brillouin law in contrast to DMS studied so far. The exciton splitting was found to be linear with the magnetization (Fig. 2),

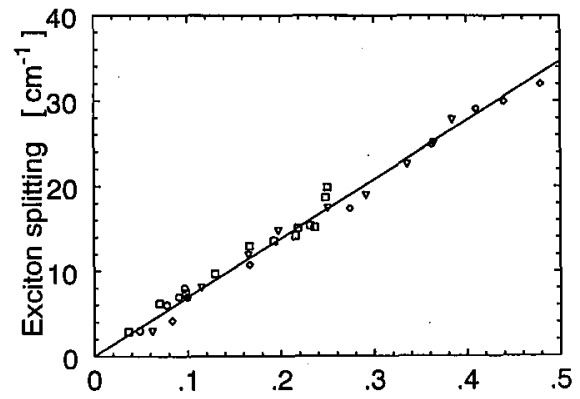

Fig. 2. Magnetization [ emu/g ]

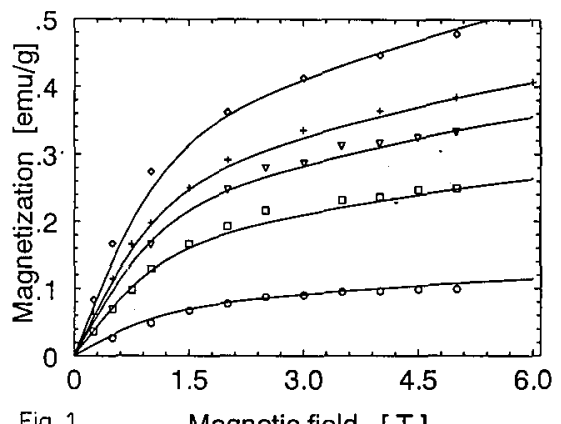

Fig. $1 \quad$ Magnetic field [ $T$ ]

Fig. 1. Magnetization of several samples of various $\mathrm{Cr}^{++}$mole fraction values, plotted vs. magnetic field. $\diamond 0.45 \%,+0.35 \%, \nabla 0.31 \%, \square 0.23 \%, \bigcirc 0.10 \%$. Lines are fits with crystal field model [4].

Fig 2. Exciton splitting $E_{\mathrm{A}}-E_{\mathrm{D}}$ vs. magnetization for four samples of various $\mathrm{Cr}^{++}$ mole fraction values. $\diamond 0.45 \%, \nabla 0.35 \%, \square 0.23 \%, \bigcirc 0.10 \%$. Line represents linear dependence with the slope $70 \mathrm{~cm}^{-1} \mathrm{~g} / \mathrm{emu}$.

similarly as it was for the other DMS $[1,4]$. Hence our results give no grounds for introducing any non-Heisenberg terms in carrier-ion interaction. Having in mind the limited magnetic ion concentration range and therefore small accuracy of our data, we do not exclude the presence of such terms.

Assuming a conventional isotropic Heisenberg-like form of the exchange interaction we get [4]

$$
E_{\mathrm{D}}-E_{\mathrm{A}}=\left(N_{0} \alpha-N_{0} \beta\right) x\langle S\rangle=\left(N_{0} \alpha-N_{0} \beta\right) m k M_{\mathrm{m}} / \mu_{\mathrm{B}}
$$

where $\langle S\rangle$ is the mean spin of $\mathrm{Cr}^{++}$ion, $\alpha=\langle S|J| S\rangle, \beta=\langle X|J| X\rangle$ are exchange integrals for the conduction and valence bands, respectively [1], $m=$ $(1-x) m_{\mathrm{Zn}}+x m_{\mathrm{Cr}}+m_{\mathrm{Se}}=m_{\text {mole }} / N_{\mathrm{Av}}$ is the mass of a DMS molecule, $\mu_{\mathrm{B}}$ is the Bohr magneton, $M_{\mathrm{m}}$ is the macroscopic magnetization and $k=\langle S\rangle /\langle M\rangle=0.53[3]$ is the ratio of the mean magnetic moment and mean spin of the Cr ion $(k=1 / 2$ for the spin only case). 
It follows from the data shown in Fig. 2 that $E_{\mathrm{A}}-E_{\mathrm{D}}=\left(70 \mathrm{~cm}^{-1} \mathrm{~g} / \mathrm{emu}\right) M_{\mathrm{m}}$ (with an accuracy of $10 \%)$, which corresponds to the exchange parameter $\left(N_{0} \alpha-\right.$ $\left.N_{0} \beta\right)=-0.67 \mathrm{eV}$. A simple way of taking into account the contribution of weak exciton components B and C would consist in multiplication of $\left(N_{0} \alpha-N_{0} \beta\right)$ by a factor $4 / 3$, producing a correct value $\left(N_{0} \alpha-N_{0} \beta\right)=0.9 \mathrm{eV}$. These values are of the same order as those found for the other DMS, typically between $1.1 \mathrm{eV}(\mathrm{CdMnTe})$ and $2.5 \mathrm{eV}(\mathrm{ZnCoSe})$, only the sign is opposite. A possible reason for the observed value of the exchange parameter is a new mechanism present in $p-d$ exchange for the $d^{4}$ electronic configuration. Roughly speaking one unoccupied $t$ orbital provides an additional exchange path, which leads to ferromagnetic $p-d$ coupling [5]. This ferromagnetic exchange may exceed the typical anti-ferromagnetic exchange (observed for Mn-, Co- and Fe-DMS [1, 2]).

\section{References}

[1] Diluted Magnetic Semiconductors, Eds. J.K. Furdyna, J. Kossut, in series Semiconductors and Semimetals, Vol. 25, Academic Press, Boston 1988; Diluted Magnetic Semiconductors, Eds. M. Averous, M. Balkanski, Plenum Press, New York 1991; Diluted Magnetic Semiconductors, Ed. M. Jain, World Scientific Press, Singapore 1991.

[2] J. Blinowski, P. Kacman, H. Przybylińska, Solid State Commun. 79, 1021 (1991).

[3] A. Twardowski, unpublished.

[4] J.A. Gaj, R. Planel, G. Fishman, Solid State Commun. 29, 435 (1979).

[5] J. Blinowski, P. Kacman, Acta Phys. Pol. A 80, 295 (1991). 